\title{
Chasing the cardiomyopathy modifier genes: Integrative computational analysis interweave the missing links in cardiomyopathy diseasome
}

\author{
Pankaj Kumar Chauhan \& R. Sowdhamini* \\ National Centre for Biological Sciences (Tata Institute of Fundamental Research), GKVK \\ Campus, Bangalore Karnataka 560065 INDIA
}

* mini@ncbs.res.in

\begin{abstract}
Background Cardiomyopathies are genetic disorders resulting in an abnormal heart phenotype due to intrinsic cardiac muscle malfunction, which leads to heart failure. These are chronic disorders contributing more than $36 \%$ of heart failure in the patients. Cardiomyopathies also show co-morbidity with other diseases that frequently affect the elderly. Apart from this, many drugs used for treating other unrelated diseases cause cardio-toxicity and lead to cardiomyopathies. Unravelling the cross-talk between them is essential for useful characterization, subtyping, and better treatment of the cardiomyopathies.
\end{abstract}

Results In this work, we applied a systems biology-based analysis to explore the associations between cardiomyopathies and other morbidities. We built a cardiomyopathy disease-disease network based on the combined biological data relevant to cardiomyopathies. We made use of publicly available genetic, non-coding RNA, drug and PPI datasets related to cardiomyopathies and network science tools to identify new modifiers genes that may have a role in cardiomyopathies. These modifier genes are further enriched using mouse phenotype data. We highlight key modifier genes that are potentially associated with cardiomyopathies. Many predicted genes link cardiomyopathies to neoplasms, metabolic and neurological disorders, some of these have been detailed as case studies in this analysis.

Conclusions Traditional one drug-one target-one disease outlook alone in drug discovery is challenging. We hereby develop an integrative approach based on primary cardiomyopathy target genes to discover new targets. These can provide clues in drug repurposing, possible off-targets and hidden genetic overlap between cardiomyopathies and other morbidities. We report potential modifier genes like $\mathrm{NOTCH}_{4}$ and SIRT1 that may be investigated for their role in cardiomyopathy and other diseases.

\section{Keywords}

Diseasome, cardiomyopathy, systems biology, protein-protein interaction, gene ontology, human phenotype ontology, network science, arrhythmogenic right ventricular cardiomyopathy, hypertrophic cardiomyopathy, dilated cardiomyopathy, modifier genes

\section{Background}

Cardiomyopathies are severe and chronic health issues across the world. These are complex heart muscle disorders compounded by genetic and environmental factors. Hypertrophic cardiomyopathy (HCM) and dilated cardiomyopathy (DCM) have an estimated prevalence of 1:500 and 1:250 in the general population [1-4]. Arrhythmogenic right ventricular cardiomyopathy (ARVC) is succeeding primary form with an estimated prevalence of one in 2000-5000 [5]. Restrictive cardiomyopathy and other forms are very rare and uncommon. Cardiomyopathies share overlapping genetic and phenotypic features with other diseases. Newer studies have revealed that several drugs, including anti-cancer, antiretroviral and antipsychotic, have shown the potential risk of cardiotoxicity and drug-induced cardiomyopathies [6-11]. Genome-wide 


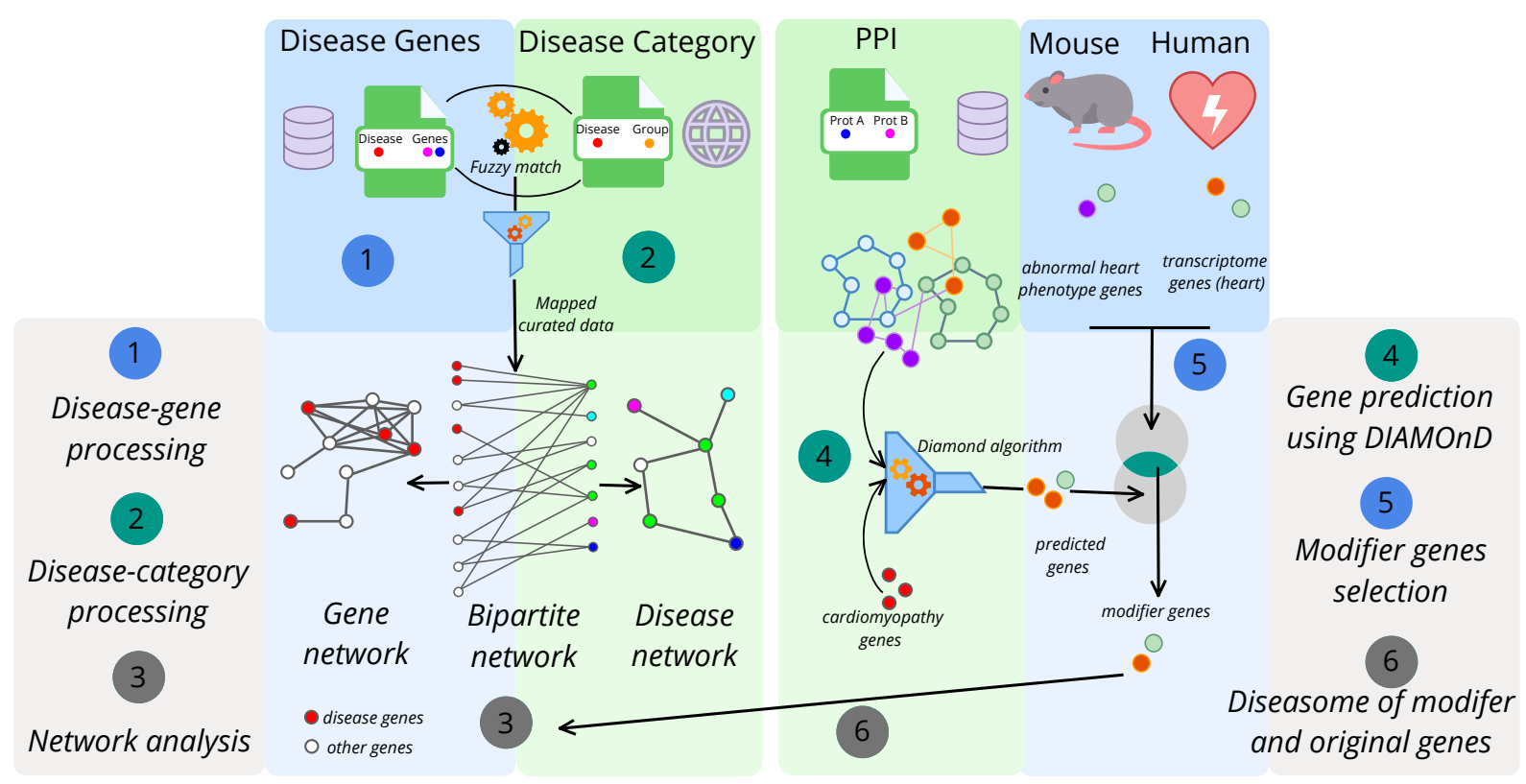

Figure 1. Schematic of modifier genes identification flowchart

association (GWAS) and candidate gene analysis approaches have reported many common genetic variants that might be one of the plausible reasons for toxicity or induced cardiomyopathy [12-15].

The emergence of network science has allowed us to understand better complex systems like social interactions, protein-protein interactions, and diseases [16-19]. Disease network analytics can apprise of disease epidemiology [20, 21]. In this direction, the first genotype-based human disease network was built based on commonly shared genes that showed the global organization of diseases and functional modules [22]. Successively, many such studies have been carried out to infer co-morbidity between diseases. This allows an eased conception of the relative risks of diseases and characteristics of their shared architecture [23-33]. Inclusion of discoveries from disease networks has found a place in therapeutic innovation and disease drug repurposing [34-39].

Cardiomyopathies have been part of many disease networks analysis [22, 25, 40]. However, no study exclusively focuses on cardiomyopathies in depth using network science to map its interaction with other diseases and common interacting genes. Sarcomere proteins are the major drivers for cardiomyopathy phenotypes. Mutations in genes like $M Y B P C 3$ and $M Y H 7$ are the most frequent and cover more than $70 \%$ of cases. Moreover, genes involved in energy metabolism and signalling also contribute to cardiomyopathies. Apart from well known genes implicated in cardiomyopathies, there is a group of other genes referred to as modifier genes that affect these disorders [41]. According to Hamilton et al. [42], 'modifier gene' refers to a genetic variant that can change the phenotypic outcome of an independent 'conditioning' variant at another locus. Modifier genes are suitable candidates for novel biomarkers, finding drug toxicity and missing links between distinct disorders. In this direction, our study is first of its kind large scale exploration of modifier genes in cardiomyopathy. It considers HCM, DCM, ARVC, RCM and all other significant forms of cardiomyopathies.

This study employs an integrative systems biology network-based comprehensive analysis to understand cardiomyopathy complexities and shared genetic architecture. To achieve that, we performed comprehensive mining of publicly available genetic, PPI, mouse phenotype, miRNA and drug targets data related to cardiomyopathies and used network tools to infer the networks. Next, we developed genetic, drug toxicity/drug repurposing and small RNA regulatory diseasome networks that will help identify novel modifiers. These genes were enriched using mouse cardiovascular abnormality gene knock out data.

We highlight essential modifier genes that link other morbidities to cardiomyopathies. The aim is to unravel new mechanisms governing the regulation of linked disease pairs. We have identified modifier genes like NOTCH4, SIRT1, SYNE1, FGFR1, ATP2A2 etc. involved in cardiomyopathies and other diseases. 


\section{Analyses}

\subsection{Genetic wiring of cardiomyopathy diseasome network genes with other pathophysiological disorders}

\subsubsection{Cardiomyopathy associated diseasome network and gene network}

Fig. 1 shows the pipeline used in the analysis. Disease-Gene data was processed in the first step, then each disease was classified into categories in step two. From theses data Bipartite network was constructed (step three). Next in step four, DIAMOnD algorithm was implemented using PPI and individual cardiomyopathy genes to predict modifier genes. Further in step five, mouse phenotype and human transcriptome data were mapped on modifier genes for validation. In step seven, we wanted to identify how these genes link cardiomyopathy to other diseases.

We found 383, 904, 3,143, and 357 gene-disease associations from OMIM, ClinVar, DisGeNet, and HumSavar respectively, by retaining only distinct diseases. The final non-redundant dataset contains 4,406 disease phenotypes. From these, cardiomyopathy genes sharing diseases were focused on for a detailed understanding of cardiomyopathy diseasome interactions. Two disorders are connected if they share at least one common gene. Further, this dataset was filtered based on each disease having at least one cardiomyopathy gene. This exclusion criterion led to the bipartite network having 146 diseases and 1,929 genes. Disease projection of bi-partite network generated genetic human disease network having 146 diseases with 1193 distinct associations (Fig. 2). These diseases belonged to many categories like metabolic, cardiovascular, neoplasms, musculoskeletal and nervous system. Many similar diseases in close vicinity were observed, such as hypertrophic cardiomyopathy and long QT syndrome or schizophrenia and bipolar disorder. Interestingly dilated cardiomyopathy and Alzheimer's disease were found to be in

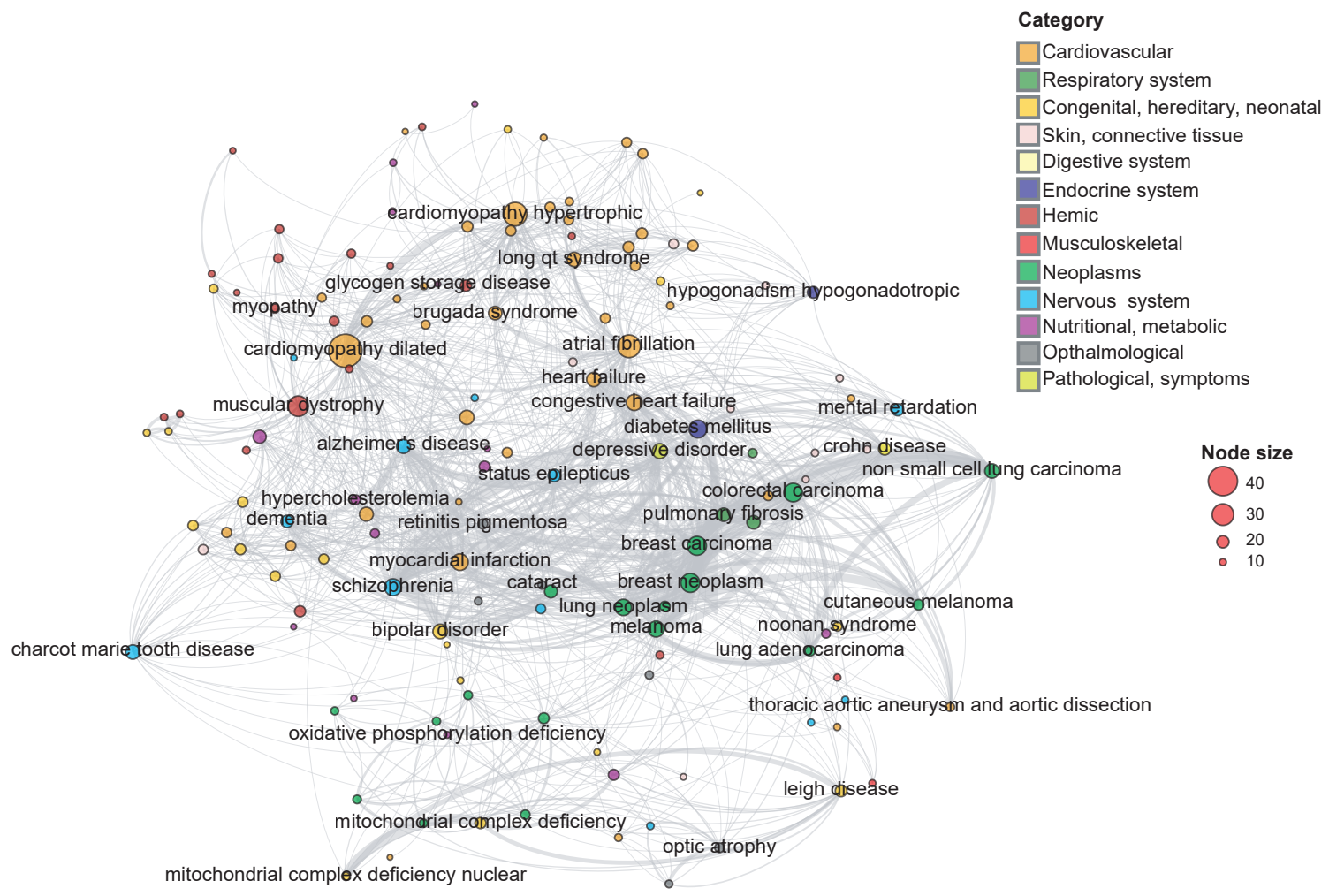

Figure 2. Cardiomyopathy specific diseasome network

In diseasome network each node means a distinct disorder, and it is colored based on the its disease class. These 12 disease classes are shown on the right. A link between disorder pair is shown in gray color. The size of each node is proportional to the number of genes in the disorder. The name of disorders with $>20$ associated genes are labelled in the network. 
a)

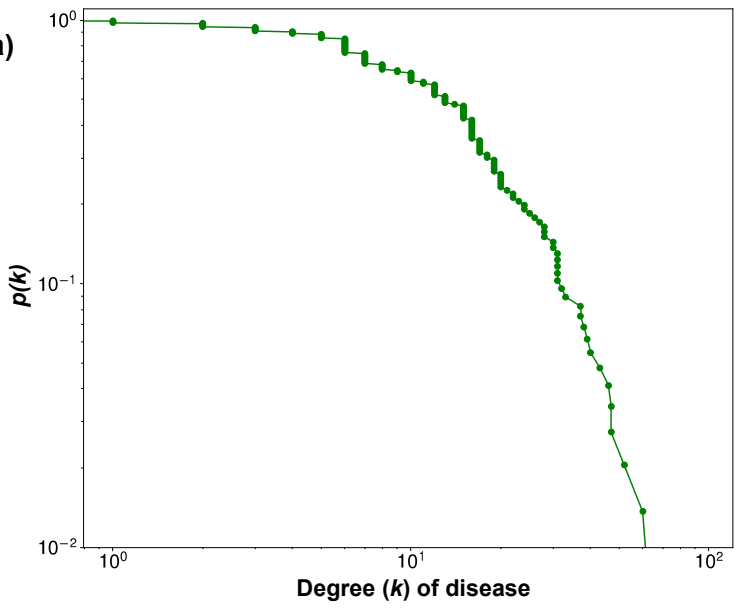

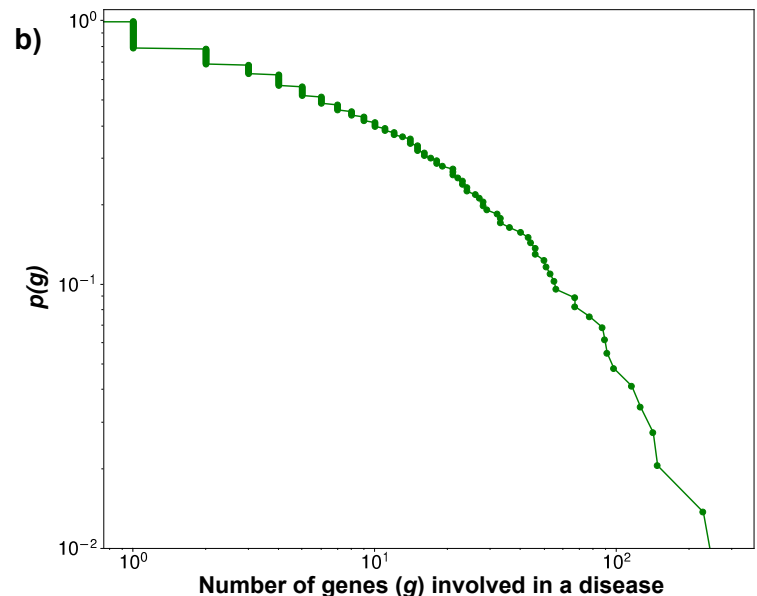

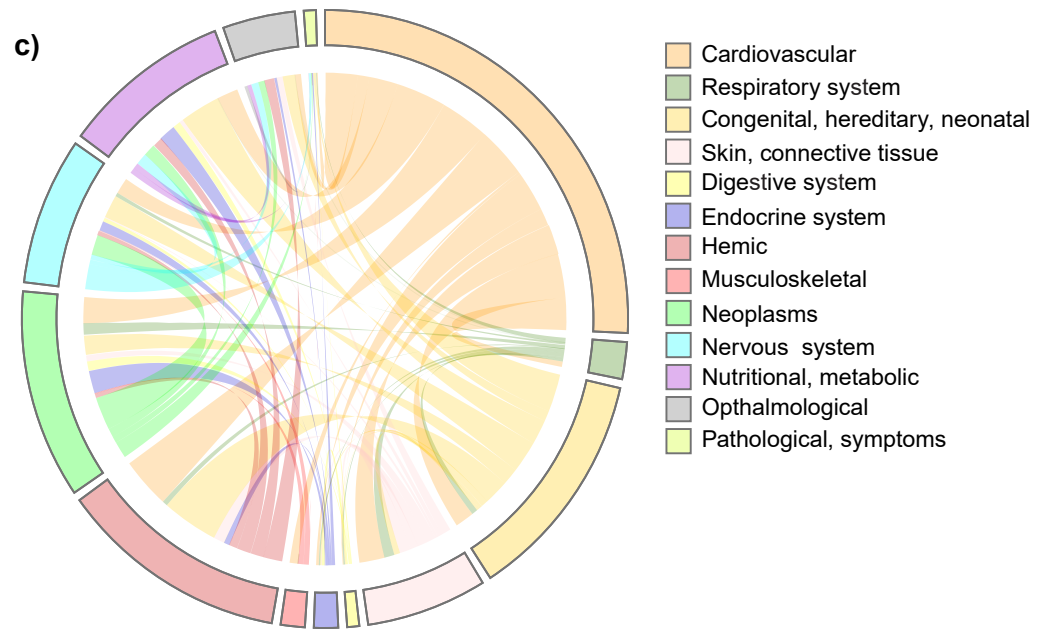

Figure 3. Cardiomyopathy diseasome network category wise interactions : Cardiomyopathy diseasome network shows complex interaction with other disorders at genetic level. a) Distribution of the degree $k$ (number of other disorders that share common genes with a particular disorder) of all disorders in cardiomyopathy diseasome. The green dots represent the logarithmically binned data. b) Distribution of the number of genes $g$ that are implicated in a particular disorder in the cardiomyopathy diseasome. c) Circosplot of disorders in each disease classes in the cardiomyopathy diseasome network. The name of the 12 disease classes are shown on the right and colored accordingly.

vicinity of each other in the network.

The degree (k) distribution of cardiomyopathy diseasome network (Fig. 3) highlights that most diseases are linked to only a few other diseases. In contrast, a few phenotypes such as dilated cardiomyopathy $(\mathrm{k}=96)$ and hypertrophic cardiomyopathy $(\mathrm{k}=63)$ are associated with many distinct disorders due to bias towards them (Table 1). The gene projection of bi-partite network for cardiomyopathy gene network consists of 1929 genes and highlights many exclusive to cardiomyopathies genes like $M Y L K 2, A L P K 3$, CSRP3, TRIM63, CALR3. On the other hand, LMNA and SCN5A were part of many diseases.

\subsubsection{Essentiality of Cardiomyopathy diseasome genes}

We used the phenotype lethality of the mouse orthologs (M-genes) and genes in the human cell viability (C-genes) data to predict the cellular essentiality of cardiomyopathy diseasome genes. A disease gene is defined as 'essential' if its mouse ortholog gene knockout leads to lethality, and also cell lines viability dataset records as essential. Essential C-genes (451) and lethal M-genes $(1,185)$ comparison analysis shows that most of the essential genes are embryonic lethal. Out of 1929 disease genes, only 54 genes were lethal in mouse and essential for cell viability (Supplement Fig. 2. file). Amongst them, only DOLK and $S D H B$ were involved in cardiomyopathies. 
Table 1. Network properties of cardiomyopathy specific diseasome network

\begin{tabular}{lccccc}
\hline Disease & Degree & DC & BC & CC & Clustering \\
\hline Dilated Cardiomyopathy & & & & & \\
Hypertrophic Cardiomyopathy & 63 & 0.434 & 0.156 & 0.62 & 0.167 \\
Atrial Fibrillation & 60 & 0.414 & 0.063 & 0.612 & 0.258 \\
Muscular Dystrophy & 52 & 0.359 & 0.055 & 0.594 & 0.268 \\
Breast Carcinoma & 47 & 0.324 & 0.021 & 0.592 & 0.398 \\
Breast Neoplasm & 47 & 0.324 & 0.022 & 0.592 & 0.404 \\
Colorectal Carcinoma & 46 & 0.317 & 0.044 & 0.578 & 0.361 \\
Diabetes Mellitus & 43 & 0.297 & 0.027 & 0.558 & 0.393 \\
Schizophrenia & 40 & 0.276 & 0.025 & 0.551 & 0.406 \\
Myocardial Infarction & 39 & 0.269 & 0.017 & 0.571 & 0.457 \\
Lung Neoplasm & 38 & 0.262 & 0.011 & 0.571 & 0.495 \\
Congestive Heart Failure & 37 & 0.255 & 0.026 & 0.556 & 0.459 \\
Melanoma & 37 & 0.255 & 0.015 & 0.569 & 0.491 \\
Depressive Disorder & 33 & 0.228 & 0.007 & 0.543 & 0.545 \\
Bipolar Disorder & 32 & 0.221 & 0.009 & 0.539 & 0.504 \\
Heart Failure & 31 & 0.214 & 0.012 & 0.503 & 0.516 \\
Non Small Cell Lung Carcinoma & 31 & 0.214 & 0.007 & 0.556 & 0.568 \\
Long QT Syndrome & 31 & 0.214 & 0.017 & 0.531 & 0.394 \\
Charcot Marie Tooth Disease & 31 & 0.214 & 0.023 & 0.533 & 0.402 \\
Left Ventricular Non Compaction & 31 & 0.214 & 0.009 & 0.533 & 0.48 \\
\hline
\end{tabular}

DC: Degree Centrality, BC: Betweenness Centrality, CC: Closeness centrality

\subsubsection{Gene-ontology of cardiomyopathy diseasome associated genes}

Genes implicated in disease are localized in specific neighbourhoods within the interactome [18]. Hence, the set of genes implicated in the same disorder should share similar cellular and functional characteristics and GO annotation. For example, HCM arises from mutations in a set of genes encoding proteins involved in sarcomeric architecture. In accordance to a previous study [22], we calculated each disorder's GO homogeneity. We observe a significant difference in Gene ontology homogeneity (GH) concerning random controls in all three branches (Fig. 4). In the random control, the same number of genes were picked randomly in the GO annotation data and GO homogeneity was measured for them. We iterated this 100 times to reach statistical significance.

\subsection{Cardiomyopathy diseasome genetic landscape links neoplasms, metabolic and musculoskeletal diseases}

\subsubsection{Cardiomyopathy exclusive diseasome associations}

The disease-disease interaction data was further reduced to include only the cardiomyopathy associations in the disease network. This focused analysis shows that these disorders belonged to cardiovascular, neoplasm, metabolic and neural categories (Fig. 5). Dominant category belonged to the cardiovascular system occupying $28.7 \%$ associations followed by musculoskeletal and congenital, each sharing $13.67 \%$ interactions. Neoplasms also contributed a surprisingly large share in cardiomyopathies interaction $(12.2 \%)$. Genetic interactions between most neoplasms and cardiomyopathies were due to RAF1 gene. Metabolic disorders were the next significant contributors (10.0\%). Among cardiomyopathies, dilated cardiomyopathy links to the most diverse disease categories. 


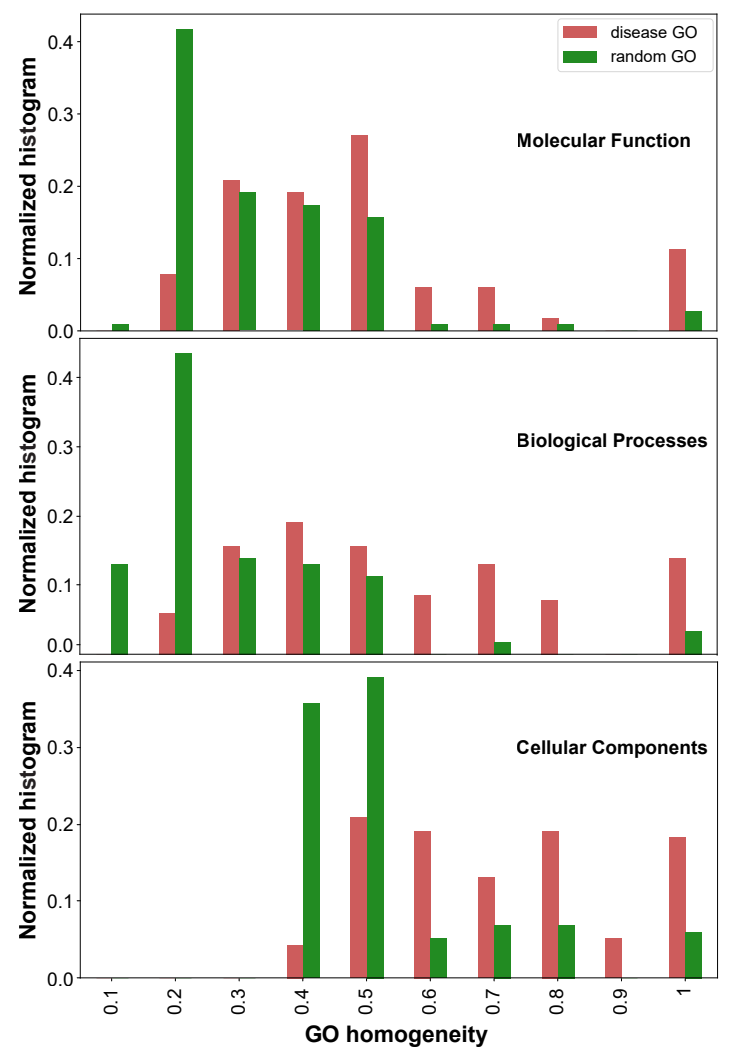

Figure 4. Gene Ontology Homogeneity: The GO homogeneity measure of cardiomyopathy associated disorders for the GO categories biological process (Left), cellular component (Center), and molecular function (Right). Red bars represent the actual disease specific histogram and the green bars show the random control, calculated for each disease by choosing the same number of genes randomly.
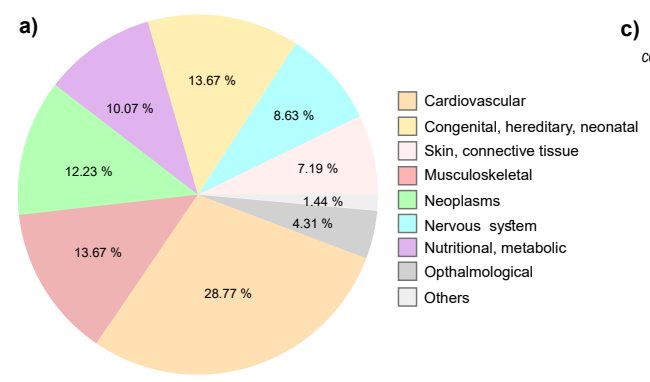

b) 70

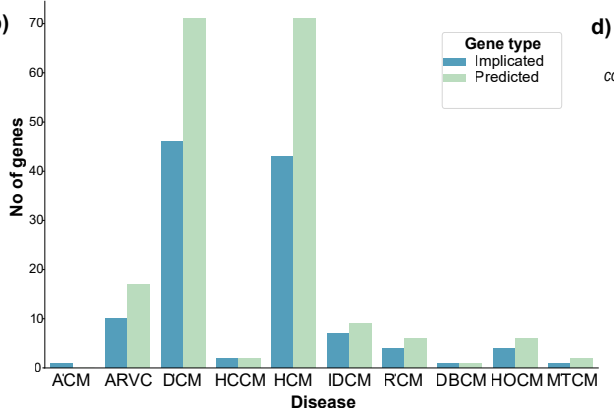

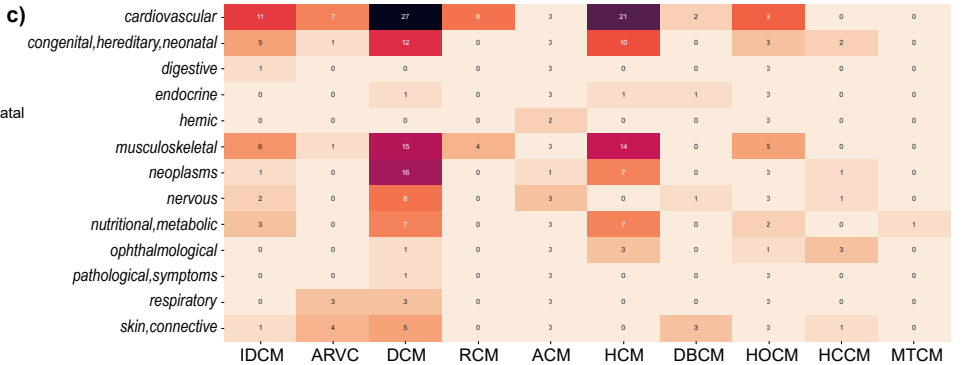

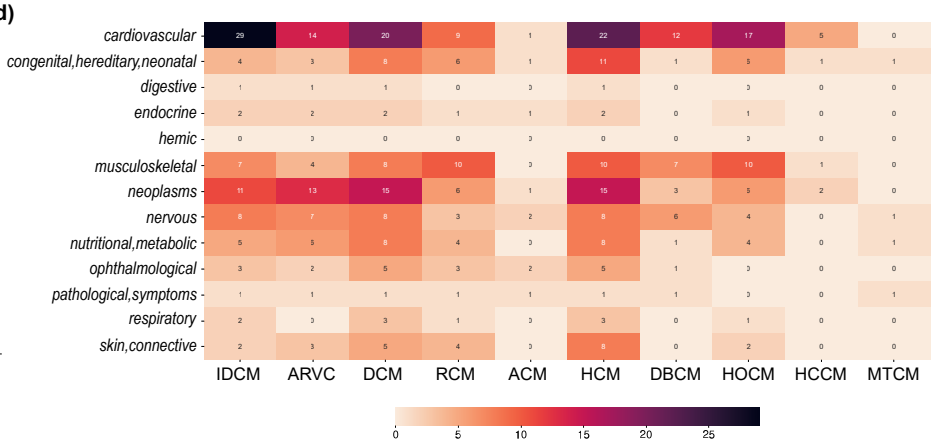

Figure 5. Characteristics of the cardiomyopathy associated disease categories. a) A pie chart showing the proportion of diseases a disorder class occupies in the cardiomyopathy diseasome network. b) A histogram showing no. of original genes and predicted new genes implicated in each cardiomyopathy. c) A heatmap showing no. of diseases in the disorder classes corresponding to each cardiomyopathy. d) A heatmap showing no. of diseases in the disorder classes corresponding to each cardiomyopathy after inclusion of modifier genes. 


\subsubsection{Identification of modifier genes using Protein-protein interaction of cardiomyopathy genes and mouse phenotype gene}

We exploited primary PPI datasets from HPRD, IntAct, MINT, CORUM and Phosphosite for comprehensive understanding of PPI [43-47]. The integrated set of PPIs contains 131,984 interactions between 14,123 proteins. Out of 1,929 disease gene products, 1,353 form a giant cluster in the PPI network, and this cluster is statistically significant compared to a random network (z-score $=18.762709 \& \mathrm{p}$-value $=$ 0.000100). To find the relationship between PPI and the number of diseases of a gene, we compared the correlation between them. There was a correlation of 0.29 (Spearman correlation) between PPI and number of diseases of a gene (Supplementary Fig. 2).

Several studies have shown that the genes associated with a specific disease tend to cluster in specific regions rather than scattered randomly, therefore, we used the DIAMOnD algorithm that exploits this pattern for finding modifier genes $[48,49]$. The DIAMOnD executes a methodological analysis of the human PPI network to unravel new disease-linked genes by exploring the connectivity significance. This algorithm was used for each cardiomyopathy separately and many new genes (50-2000) were predicted compared to the original implicated genes. We used international mouse phenotypic consortium (IMPC) cardiovascular abnormality data for identification of modifier genes. Mapping of these genes to human orthologs (HGNC) identified 615 genes. The DIAMoND algorithm predicted genes were mapped to these 615 genes for identification of cardiomyopathy specific modifier genes. This stringent filtering increases the robustness of the network (Table 2).

\subsubsection{Modifier genes as a medium to associate cardiomyopathies to other morbidities}

Based on implicated genes and predicted genes, we categorized disease links into two categories: a) established associations and b) predicted associations from modifier genes (for more details see Supplementary Table 1). Apart from the original 69 cardiomyopathies associations to other disorders, additional 139 interactions were predicted using modifier genes as linkage. 
bioRxiv preprint doi: https://doi.org/10.1101/2021.02.26.433014; this version posted March 11, 2021. The copyright holder for this preprint (which was not certified by peer review) is the author/funder. All rights reserved. No reuse allowed without permission.

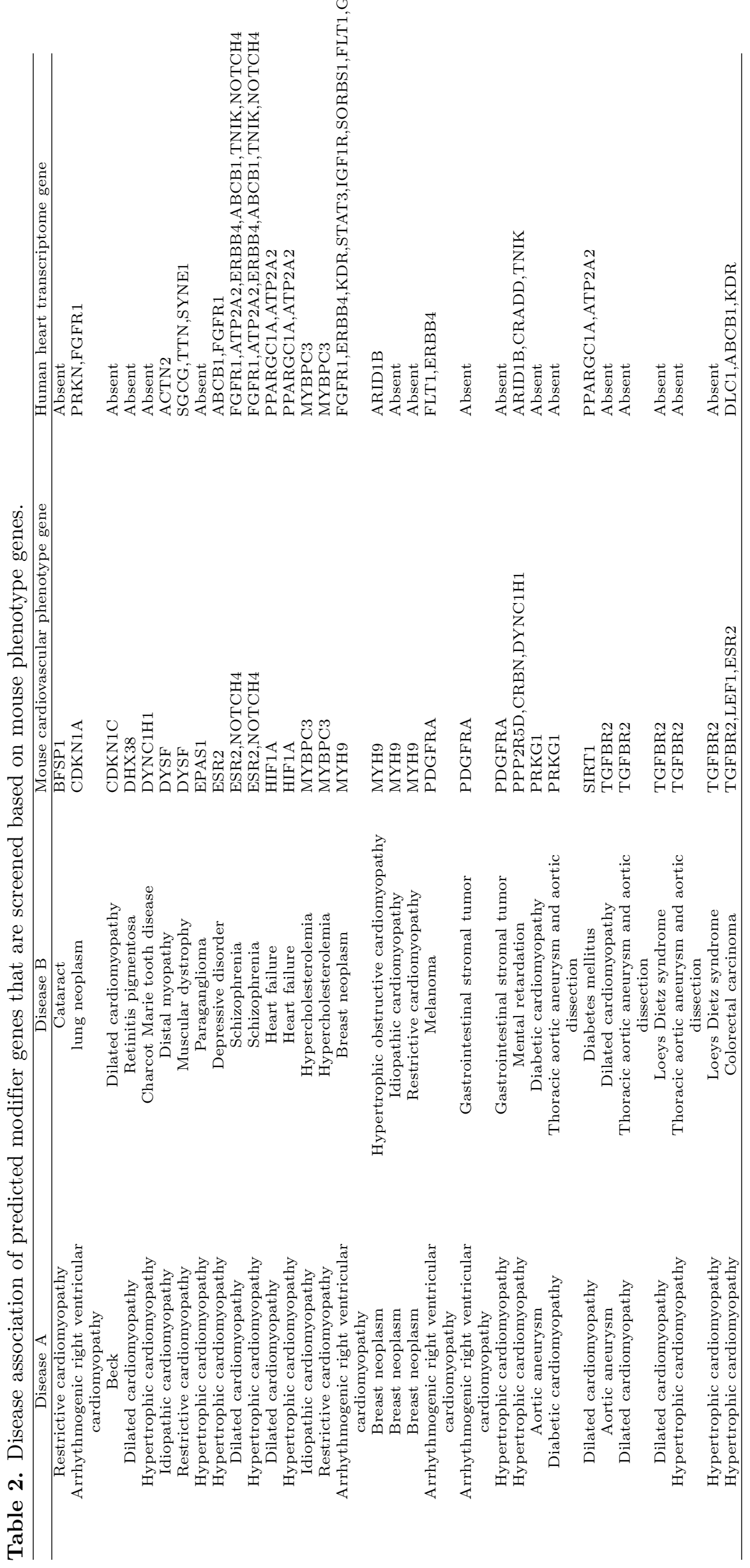




\subsubsection{Drug-targets and miRNA datasets as a medium to associate cardiomyopathies to other morbidities}

We conceptualized the drug-based disease network by therapeutic links among diseases that may convey the underlying similarities among diseases. Drug based cardiomyopathy diseasome network consists of 391 nodes and 18994 edges. For visualization we focused on only cardiomyopathy associations (Supplementary Fig. 3). Neoplasms and nervous system disorders dominate this network. Apart from intragroup connectivity, many diseases have strong inter category disease connectivity. Such resemblance could convey related biological pathways, co-morbidities, or new indications for drugs.

There is an increasingly new interest in post-transcriptional regulation's molecular mechanism, emphasizing non-coding RNAs in cardiomyopathies [50-52]. We visualized cardiomyopathy specific miRNA diseasome network by retaining miRNAs whose at least one target gene was present in cardiomyopathy gene list. miRNA associated cardiomyopathy diseasome network consists of 935 nodes and 187479 edges. To highlight cardiomyopathy specific interactions, we show only these in visualization (Supplementary Fig. 4)

\subsection{Cardiomyopathy modifier genes case studies}

Our study predicts many novel modifier genes that have been implicated in schizophrenia, neoplasms, Alzheimer's, hypogonadism hypogonadotropic and other diseases. We performed a literature search to assess the biological relevance of the results. Twenty-two modifier genes showed cardiovascular abnormalities in mouse phenotypes. In contrast, forty-nine modifier genes were expressed in heart tissue, as mentioned in [53]. ATP2A2 [54], IGF1R [55], FGFR1 [56], PDGFRA [57], CDKN1C [58], SYNE1 [59, 60], NOTCH4 [61], SIRT1 [62] etc. ATP2A2 gene was found to link acrokeratosis verruciformis disease to HCM and DCM. IGF1R was found to associate Alzheimer's disease to ARVC and DCM. Bipolar disorders associated gene $S Y N E 1$ was observed in idiopathic cardiomyopathy. Disease-associated with $\mathrm{NOTCH}_{4}$ includes schizophrenia and may be linked to HCM and DCM. Similarly, SIRT1 is implicated in diabetes mellitus and may be a modifier in DCM.

SIRT1 is a multi-functional protein involved in controlling DNA repair, tissue regeneration, cell survival, inflammation, signalling, and circadian rhythms [63, 63-65]. We predicted link between cardiomyopathy and diabetes mellitus with SIRT1 as a common gene. Previous works[66] have shown SIRT1 in protecting pancreatic $\beta$-cells from cytokine toxicity. In another study, Lan Cui et al.[67] indicated that EPO might activate SIRT1 to enhance mitochondrial function and protect against DOX-induced cardiotoxicity. One of the latest studies demonstrated that Resveratrol-mediated SIRT1 activation attenuated tesaglitazar-induced cardiac dysfunction [68].

\section{Discussion}

Cardiomyopathies, are not restricted to sarcomeric protein-coding genes. Involvement of metabolic, signalling associated, and other modifier genes also influence these phenotypes. Many studies have shown that disease co-morbidities arise due to shared genes [23, 24]. These shared genes may not be directly associated; therefore, an integrative, disease-specific network can unravel these hidden key genes to gain more insights into the disease co-morbidity and drug toxicity. In this study, we present cardiomyopathy focused diseasome network that examines all major cardiomyopathies in detail. In our approach, we use systems biology principles to link cardiomyopathies to other diseases or morbidities through shared genes, drug targets, and miRNAs that provide insights about modifier genes, drug toxicity, and non-genetic regulation. Genetic data (OMIM, ClinVar, DisGeNet and HumSavar) provide a credible source of disease and its genes. We present cardiomyopathy exclusive diseasome with 146 disorders and 1,929 associated genes that show many pathophysiological disorders from neurological, musculoskeletal, metabolic and neoplasms. Human cell viability and mouse phenotype lethality data give an insight into the nature of the genes involved in these diseases. Our analysis shows that most genes involved in the diseasome are not essential for cell viability and are also connected to a few diseases only. Protein-protein interaction data (HPRD, MINT, IntAct, CORUM and Phosphosite) outlines functional connectivity amongst genes. It helps identify close interactors/related genes (directly interacting gene products in PPI) genes implicated as modifiers. PPI and mouse phenotype data can fill the missing link between two non-obvious diseases. Similarly, gene ontology (GO) terms convey the molecular level information genes in diseases. Majority of 
genes in disease are involved in the same biological process or are part of the same cellular component. We used mouse phenotype data and heart tissue transcriptome data to validate modifiers' role in cardiovascular abnormalities. This analysis led to the identification of new modifiers linking cardiomyopathies to other disorders. Our integrated approach demonstrates the power of network study to find novel modifier genes linking distinct disorders. SIRT1, SYNE1, NOTCH4, ATP2A2 and IGF1R look promising modifiers for cardiomyopathies. Later we looked at common drug and miRNAs that have gene targets in two different diseases. Drug based diseasome networks highlight the therapeutic interconnectivity of different diseases. Many of these diseases belong to different categories. The separate disease-disease network was constructed for miRNA set. Many studies demonstrate that microRNAs carry out similar functions by targeting either the same gene or functionally related genes [69, 70]. miRNA associated diseasome network will be resourceful in deciphering the roles of mRNAs in disease associations. Drug and miRNA target diseasome network demonstrate and inter connectivity of cardiomyopathy to neoplasms, nervous system and other diseases.

This study's primary limitation is the initial data noise, which may remain in the outcome even after processing. Further, the disease associations are based on either common shared genes, drug or miRNA only and may miss physical co-morbidity. In future, we would like to study structural and in-silico point mutational analysis of these modifiers in cardiomyopathies.

\section{Potential implications}

Our study's approach to identify links between cardiomyopathies and other pathophysiological disorders may also be extended to other disease-specific network. The predicted modifier genes in this study can be taken up for experimental and clinical validations.

\section{Methods}

\subsection{Diseases-gene association data sources}

Genes and diseases association data were downloaded from OMIM(v2018), ClinVar(2020), HumSaVar(2020) and DisGeNet(2020) datasets. Custom python scripts were used for the pre-processing of each dataset. From the OMIM dataset, only '(3)' marked diseases were selected for curated data. Similarly, in DisGeNet, diseases with a score above 0.4 were considered. HGNC gene symbols were assigned to each gene for a consistent and more accurate name. Synonymic or alternative gene names were reduced to the HGNC gene primary symbol, as reported in HGNC (June 2020 release). All disease-gene datasets were merged and manually curated for a comprehensive non-redundant disease-gene data. Similar diseases, disease sub-types or the same disease with different names were merged (d-2 hydroxyglutaric aciduria and l-2 hydroxyglutaric aciduria were merged together and named hydroxyglutaric aciduria).

\subsection{Diseases-category association data sources}

MeSH 2020 (2020), GARD (2020), and Literature [22] data were integrated for the categorization of diseases. When mapping MeSH terms, we only used the portions of the vocabulary from Diseases $[\mathrm{C}]$. A custom python script was used to process the disease phenotype data. The category names were assigned according to significant organ systems based on MeSH 2020, and similar types were merged, thus restricting the number of the category to 12. A different 'multiple' group was created to cater to diseases belonging to multiple categories. If a disorder was part of many categories, the dominant type was assigned. In the case of multiple dominant categories, it was assigned to the 'multiple' category. Some diseases may be misassigned to a category as categorization of diseases is still a research problem.

\subsection{Disease-Gene-category Association}

Fuzzy pattern matching algorithm, was used for Disease-Gene-Category analysis. It assigned the best category for disease using partial and full ratio match from fuzzy logic using a cut-off of $70 \%$ similarity 
for the safe category assignment. Assigned categories were further manually curated for any discrepancies. Around $30 \%$ of diseases were randomly picked for manual curation. Only those disease, where at least one cardiomyopathy gene was present, were retained for further analysis.

\subsection{Diseasome network and Gene network construction}

The bipartite network between disease and genes was constructed from the previous data with disorders as one category of nodes and genes another type. A disease-gene pair were linked if the gene was part of that disease. Then cardiomyopathy diseasome network was obtained by projecting the network on disease nodes. The degree $(\mathrm{k})$ distribution of cardiomyopathy diseasome was calculated using networkx. Node size is proportional to the number of genes in the disease, and edge width is proportional to the common in both diseases. Nodes are coloured according to categories of diseases. Similarly, the cardiomyopathy gene network was constructed by projecting the bipartite network on gene nodes. This whole analysis was done using networkx module in python.

\subsection{Gene ontology of diseasome}

Molecular function, cellular component and biological processes for disease genes were obtained from the UniProt database. For this, all genes in the full diseasome were mapped to UniProt gene symbol. In this analysis, 4923 out of 4995 disease genes mapped to UniProt primary gene map. The GO analysis was applied to each disease linked to cardiomyopathies only. The statistical significance of the dominant GO in each disorder was compared against the random condition. In this, the GO homogeneity $(\mathrm{GH})$ of each disorder was measured as the maximum fraction of genes in the same disease with the same GO terms. It is defined as

$$
G H_{i}=\max _{j} \frac{n_{j i}}{n_{i}}
$$

wherein this case $n_{i}$ denotes the number of genes in the disorder $i$ that have any GO annotations, and $n_{j i}$ the number of genes with a specific GO term $j . G H_{i}$ was calculated separately for biological process $(\mathrm{BP})$, molecular function $(\mathrm{MF})$, and cellular component (CC).

\subsection{Essential and non-essential genes analysis}

Cell lines viability and mouse phenotype lethality data used in the previous study was used for essential and nonessential genes classification [71]. Data was processed, and cardiomyopathy diseasome associated genes were mapped to it.

\subsection{Protein-protein interaction network analysis}

Human PPI data (HPRD, MINT, and IntAct ) were integrated with other interactions (protein complex and kinase substrate) from CORUM and Phosphosite databases for comprehensive interaction data. Cardiomyopathy diseasome genes were mapped on this comprehensive PPI data to find a diseasome specific PPI network.

\subsection{Gene prediction and cardiovascular phenotype validation}

Fifty times the original genes implicated in each cardiomyopathy were predicted from PPI using the diamond algorithm. Mouse cardiovascular abnormality phenotype genes were downloaded from IMPC. From cardiovascular data, heart-specific phenotypes genes were filtered manually for further analysis.Mouse genes and heart tissue-specific transcriptome genes were used as validation for predicted genes from the PPI network. If any of these genes involved other diseases, we called them putative candidates linking both diseases. 


\subsection{Drug-target and MiRNA based disease interactome analysis}

Drugs and its targets data were downloaded from DrugBank 2018 and DrugCentral 2020 [72, 73]. The files were parsed using a python script, and only human-specific data were used for further analysis. This data was used for finding drug target genes linking two diseases. We subsequently constructed a hypothetical human diseasome based on common miRNA targeting genes from different diseases. Diseasome network was constructed to incorporate putative miRNA targets in cardiomyopathy diseases.

\section{$5 \quad$ Availability of supporting data and materials}

Scripts and data are available upon request to authors.

\section{Additional Files}

Supplementary Materials and Figures. Further details regarding gene network, essential genes, modifier genes in each cardiomyopathy and full data set table.

Supplementary Fig. 1. Gene network

Supplementary Fig. 2. a) Correlation betwwen PPI number and number of diseases caused by a gene. b) Frequency of diseases caused by a gene. c) Venn diagram of essential and lethal genes. d) Degree connectivity of essential v/s non-essential genes

Supplementary Fig. 3 Drug target diseasome network

Supplementary Fig. 4 miRNA target diseasome network

Supplementary Table S1. Modifier genes full table

\section{Declarations}

\subsection{List of abbreviations}

ACM: Amyloid Aardiomyopathy ARVC: Arrhythmogenic Right Ventricular Cardiomyopathy CORUM: The comprehensive resource of mammalian protein complexes

DBCM: Diabetic Cardiomyopathy

DCM: Dilated Cardiomyopathy

GARD: The Genetic and Rare Diseases

GWAS: Genome-wide association

HCCM: Histocoid Cardiomyopathy

HCM: Hypertrophic Cardiomyopathy

HGNC: HUGO Gene Nomenclature Committee

HOCM: Hypertrophic Obstructive Cardiomyopathy

HPO: human Phenotype Ontology

HPRD: The Human Protein Reference Database

IDCM: Idiopathic Cardiomyopathy

MeSH : Medical Subject Headings

MINT: The Molecular INTeraction Database

MTCM: Mitochondrial Cardiomyopathy

OMIM: Online Mendelian Inheritance in Man

PPI: Protein-Protein Interaction

RCM: Restrictive Cardiomyopathy

\subsection{Ethical Approval (optional)}

Not applicable 


\subsection{Consent for publication}

Not applicable

\subsection{Competing Interests}

The authors declare that they have no competing interests.

\subsection{Funding}

The authors would like to thank NCBS (TIFR) for infrastructural facilities. RS acknowledges funding and support provided by JC Bose Fellowship (SB/S2/JC-071/2015) from Science and Engineering Research Board, India and Bioinformatics Centre Grant funded by Department of Biotechnology, India (BT/PR40187/BTIS/137/9/2021.

\subsection{Author's Contributions}

PKC and RS conceptualized the study. PKC collected data, performed data analysis and visualization, developed and implemented the codes. PKC and RS wrote the final draft of manuscript.

\section{Acknowledgements}

We would like to thank Murugavel for technical help, whenever required. PKC would like to thank NCBS-TIFR for the fellowship.

\section{References}

[1] Antunes MdO, Scudeler TL, Hypertrophic cardiomyopathy. Elsevier Ireland Ltd; 2020.

[2] Maron BJ, Maron MS, Maron BA, Loscalzo J, Moving Beyond the Sarcomere to Explain Heterogeneity in Hypertrophic Cardiomyopathy: JACC Review Topic of the Week. Elsevier USA; 2019. https://doi.org/10.1016/j.jacc.2019.01.061.

[3] Hershberger RE, Hedges DJ, Morales A, Dilated cardiomyopathy: The complexity of a diverse genetic architecture. Nature Publishing Group; 2013.

[4] Peters S, Johnson R, Birch S, Zentner D, Hershberger RE, Fatkin D, Familial Dilated Cardiomyopathy. Elsevier Ltd; 2020.

[5] Corrado D, Link MS, Calkins H. Arrhythmogenic Right Ventricular Cardiomyopathy. New England Journal of Medicine 2017 jan;376(1):61-72. http://www .nejm.org/doi/10. 1056/NEJMra1509267.

[6] Gupta A, Mandala A, Wee Y. Drug-Induced Cardiomyopathy: An Institutional Experience. Heart, Lung and Circulation 2017 jan;26:S132-S133. http://dx.doi.org/10.1016/j.hlc.2017.06.202.

[7] Albakri A. Drugs-related cardiomyopathy: A systematic review and pooled analysis of pathophysiology, diagnosis and clinical management. Review Article Internal Medicine and Care 2019;3:1-19.

[8] Wu AH, Cardiotoxic drugs: Clinical monitoring and decision making; 2008.

[9] Barish R, Gates E, Barac A, Trastuzumab-Induced Cardiomyopathy. W.B. Saunders; 2019. https://pubmed.ncbi.nlm.nih.gov/31587782/.

[10] Bansal N, Adams MJ, Ganatra S, Colan SD, Aggarwal S, Steiner R, et al. Strategies to prevent anthracycline-induced cardiotoxicity in cancer survivors. Cardio-Oncology 2019 dec;5(1):1-22. https://link. springer.com/articles/10.1186/s40959-019-0054-5 https://link.springer.com/article/10.1186/s40959-019-0054-5. 
[11] Garcia-Pavia P, Kim Y, Restrepo-Cordoba MA, Lunde IG, Wakimoto H, Smith AM, et al. Genetic Variants Associated With Cancer Therapy-Induced Cardiomyopathy. Circulation 2019 jul;140(1):3141 .

[12] Wells QS, Veatch OJ, Fessel JP, Joon AY, Levinson RT, Mosley JD, et al. Genome-wide association and pathway analysis of left ventricular function after anthracycline exposure in adults. Pharmacogenetics and Genomics 2017;27(7):247-254. https://pubmed.ncbi.nlm.nih.gov/28542097/.

[13] Wojnowski L, Kulle B, Schirmer M, Schlüter G, Schmidt A, Rosenberger A, et al. $\mathrm{NAD}(\mathrm{P}) \mathrm{H}$ oxidase and multidrug resistance protein genetic polymorphisms are associated with doxorubicin-induced cardiotoxicity. Circulation 2005 dec;112(24):3754-3762. https://pubmed.ncbi.nlm.nih.gov/16330681/.

[14] Visscher H, Ross CJD, Rassekh SR, Sandor GSS, Caron HN, van Dalen EC, et al. Validation of variants in $<\mathrm{i}>\mathrm{SLC} 28 \mathrm{~A} 3</ \mathrm{i}>$ and $<\mathrm{i}>\mathrm{UGT} 1 \mathrm{~A} 6</ \mathrm{i}>$ as genetic markers predictive of anthracycline-induced cardiotoxicity in children. Pediatric Blood \& Cancer 2013 aug;60(8):1375-1381. http://doi.wiley.com/10.1002/pbc. 24505.

[15] Blanco JG, Leisenring WM, Gonzalez-Covarrubias VM, Kawashima TI, Davies SM, Relling MV, et al. Genetic polymorphisms in the carbonyl reductase 3 gene CBR3 and the NAD $(\mathrm{P}) \mathrm{H}$ :quinone oxidoreductase 1 gene NQ01 in patients who developed anthracycline-related congestive heart failure after childhood cancer. Cancer 2008 jun;112(12):2789-2795. https://pubmed.ncbi.nlm.nih.gov/18457324/.

[16] Barabási AL, Oltvai ZN, Network biology: Understanding the cell's functional organization. Nature Publishing Group; 2004. www.nature.com/reviews/genetics.

[17] Hidalgo CA, Blumm N, Barabási AL, Christakis NA. A Dynamic Network Approach for the Study of Human Phenotypes. PLoS Computational Biology 2009 apr;5(4). https://pubmed.ncbi.nlm.nih.gov/19360091/.

[18] Barabási AL, Gulbahce N, Loscalzo J, Network medicine: A network-based approach to human disease. Nature Publishing Group; 2011. www.nature.com/reviews/genetics.

[19] Bliss CA, Kloumann IM, Harris KD, Danforth CM, Dodds PS. Twitter reciprocal reply networks exhibit assortativity with respect to happiness. Journal of Computational Science 2012 sep;3(5):388 397.

[20] Feldman I, Rzhetsky A, Vitkup D. Network properties of genes harboring inherited disease mutations. Proceedings of the National Academy of Sciences of the United States of America 2008 mar;105(11):4323-4328. www.pnas.orgcgidoi10.1073pnas.0701722105.

[21] Zanzoni A, Soler-López M, Aloy P, A network medicine approach to human disease. FEBS Lett; 2009. https://pubmed.ncbi.nlm.nih.gov/19269289/.

[22] Goh KI, Cusick ME, Valle D, Childs B, Vidal M, Barabási AL. The human disease network. Proceedings of the National Academy of Sciences of the United States of America 2007 may;104(21):8685-8690. www. pnas.org/cgi/content/full/.

[23] Rzhetsky A, Wajngurt D, Park N, Zheng T. Probing genetic overlap among complex human phenotypes. Proceedings of the National Academy of Sciences of the United States of America 2007 jul;104(28):11694-11699. www . pnas .org/cgi/content/full/.

[24] Divo MJ, Casanova C, Marin JM, Pinto-Plata VM, De-Torres JP, Zulueta JJ, et al. COPD comorbidities network. European Respiratory Journal 2015 sep;46(3):640-650. https://pubmed.ncbi.nlm.nih.gov/26160874/.

[25] Halu A, De Domenico M, Arenas A, Sharma A. The multiplex network of human diseases. npj Systems Biology and Applications 2019 dec;5(1):1-12. https://doi.org/10.1038/s41540-019-0092-5.

[26] Cassidy-Bushrow AE, Bielak LF, Sheedy PF, Turner ST, Chu JS, Peyser PA. Shared genetic architecture in the relationship between adult stature and subclinical coronary artery atherosclerosis. Atherosclerosis 2011 dec;219(2):679-683. 
[27] Lee DS, Park J, Kay KA, Christakis NA, Oltvai ZN, Barabási AL. The implications of human metabolic network topology for disease comorbidity. Proceedings of the National Academy of Sciences of the United States of America 2008 jul;105(29):9880-9885. https://www.pnas.org/content/105/29/9880 https://www . pnas.org/content/105/29/9880. abstract.

[28] Li L, Ruau DJ, Patel CJ, Weber SC, Chen R, Tatonetti NP, et al. Disease risk factors identified through shared genetic architecture and electronic medical records. Science Translational Medicine 2014 apr;6(234):234ra57-234ra57. https://stm.sciencemag.org/content/6/234/234ra57 https://stm.sciencemag.org/content/6/234/234ra57.abstract.

[29] Li YR, Li J, Zhao SD, Bradfield JP, Mentch FD, Maggadottir SM, et al. Meta-analysis of shared genetic architecture across ten pediatric autoimmune diseases. Nature Medicine 2015 sep;21(9):1018 1027. https://pubmed.ncbi.nlm.nih.gov/26301688/.

[30] Zhou X, Menche J, Barabási AL, Sharma A. Human symptoms-disease network. Nature Communications 2014 jun;5(1):1-10. www.nature.com/naturecommunications.

[31] Glicksberg BS, Li L, Badgeley MA, Shameer K, Kosoy R, Beckmann ND, et al. Comparative analyses of population-scale phenomic data in electronic medical records reveal race-specific disease networks. Bioinformatics 2016 jun;32(12):i101-i110. https://academic.oup.com/bioinformatics/article-lookup/doi/10.1093/bioinformatics/btw282.

[32] Blair DR, Lyttle CS, Mortensen JM, Bearden CF, Jensen AB, Khiabanian H, et al. A nondegenerate code of deleterious variants in mendelian loci contributes to complex disease risk. Cell 2013 sep;155(1):70-80.

[33] Barrenas F, Chavali S, Holme P, Mobini R, Benson M. Network Properties of Complex Human Disease Genes Identified through Genome-Wide Association Studies. PLoS ONE 2009;4(11).

[34] Dudley JT, Sirota M, Shenoy M, Pai RK, Roedder S, Chiang AP, et al. Computational repositioning of the anticonvulsant topiramate for inflammatory bowel disease. Science Translational Medicine 2011 aug;3(96):96ra76-96ra76. https://stm.sciencemag.org/content/3/96/96ra76 https://stm.sciencemag.org/content/3/96/96ra76. abstract.

[35] Schadt EE, Friend SH, Shaywitz DA. A network view of disease and compound screening. Nature Reviews Drug Discovery 2009;8(4):286-295. https://www.nature.com/articles/nrd2826.

[36] Shameer K, Readhead B, T Dudley J. Computational and Experimental Advances in Drug Repositioning for Accelerated Therapeutic Stratification. Nature Reviews Drug Discovery 2015;15(4):5 20. https : //www. eurekaselect. com.

[37] Oti M, Huynen MA, Brunner HG, Phenome connections. Elsevier Current Trends; 2008.

[38] Vanhaelen Q, Mamoshina P, Aliper AM, Artemov A, Lezhnina K, Ozerov I, et al., Design of efficient computational workflows for in silico drug repurposing. Elsevier Ltd; 2017. https://pubmed.ncbi.nlm.nih.gov/27693712/.

[39] Haslam B, Perez-Breva L. Learning disease relationships from clinical drug trials. Journal of the American Medical Informatics Association 2017 jan;24(1):13-23. https://academic.oup.com/jamia/article/24/1/13/2631432.

[40] Haidar MN, Islam MB, Chowdhury UN, Rahman MR, Huq F, Quinn JMW, et al. Network-based computational approach to identify genetic links between cardiomyopathy and its risk factors. IET Systems Biology 2020 apr;14(2):75-84. https://pubmed.ncbi.nlm.nih.gov/32196466/.

[41] Chen Y, Xu F, Munkhsaikhan U, Boyle C, Borcky T, Zhao W, et al. Identifying modifier genes for hypertrophic cardiomyopathy. Journal of Molecular and Cellular Cardiology 2020 jul;144:119-126. http://www.jmcc-online.com/article/S0022282820301401/fulltext http://www.jmcc-online.com/article/S0022282820301401/abstract https://www .jmcc-online.com/article/S0022-2828(20)30140-1/abstract. 
[42] Hamilton BA, Yu BD. Modifier Genes and the Plasticity of Genetic Networks in Mice. PLoS Genetics 2012 apr;8(4):e1002644. https://dx.plos.org/10.1371/journal.pgen. 1002644.

[43] Keshava Prasad TS, Goel R, Kandasamy K, Keerthikumar S, Kumar S, Mathivanan S, et al. Human Protein Reference Database - 2009 update. Nucleic Acids Research 2009;37(SUPPL. 1). https://pubmed.ncbi.nlm.nih.gov/18988627/.

[44] Orchard S, Ammari M, Aranda B, Breuza L, Briganti L, Broackes-Carter F, et al. The MIntAct project - IntAct as a common curation platform for 11 molecular interaction databases. Nucleic Acids Research 2014 jan;42(D1). https://pubmed.ncbi.nlm.nih.gov/24234451/.

[45] Licata L, Briganti L, Peluso D, Perfetto L, Iannuccelli M, Galeota E, et al. MINT, the molecular interaction database: 2012 Update. Nucleic Acids Research 2012 jan;40(D1). https://pubmed.ncbi.nlm.nih.gov/22096227/.

[46] Hornbeck PV, Chabra I, Kornhauser JM, Skrzypek E, Zhang B. PhosphoSite: A bioinformatics resource dedicated to physiological protein phosphorylation. Proteomics 2004 jun;4(6):1551-1561. https://pubmed.ncbi.nlm.nih.gov/15174125/.

[47] Giurgiu M, Reinhard J, Brauner B, Dunger-Kaltenbach I, Fobo G, Frishman G, et al. CORUM: The comprehensive resource of mammalian protein complexes - 2019. Nucleic Acids Research 2019 jan;47(D1):D559-D563. http://mips.helmholtz-muenchen.de/corum/.

[48] Ghiassian SD, Menche J, Barabási AL. A DIseAse MOdule Detection (DIAMOnD) Algorithm Derived from a Systematic Analysis of Connectivity Patterns of Disease Proteins in the Human Interactome. PLOS Computational Biology 2015 apr;11(4):e1004120. https://dx.plos.org/10.1371/journal.pcbi.1004120.

[49] Fiscon G, Conte F, Farina L, Paci P, Network-based approaches to explore complex biological systems towards network medicine. MDPI AG; 2018. https://pubmed.ncbi.nlm.nih.gov/30200360/.

[50] Charles S, Natarajan J. Two way network construction and analysis of mRNA, miRNA and lncRNA reveals critical regulators and regulatory modules in cardiovascular diseases. Genes and Genomics 2020 aug;42(8):855-867. https ://doi.org/10.1007/s13258-020-00952-6.

[51] Le TD, Zhang J, Liu L, Li J. Computational methods for identifying miRNA sponge interactions. Briefings in Bioinformatics 2017 jul;18(4):577-590. https://pubmed.ncbi.nlm.nih.gov/27273287/.

[52] Jiang Q, Hao Y, Wang G, Juan L, Zhang T, Teng M, et al. Prioritization of disease microRNAs through a human phenome-microRNAome network. BMC Systems Biology 2010 may;4(SUPPL. 1):S2. https://bmcsystbiol.biomedcentral.com/articles/10.1186/1752-0509-4-S1-S2.

[53] Tucker NR, Chaffin M, Fleming SJ, Hall AW, Parsons VA, Bedi KC, et al. Transcriptional and Cellular Diversity of the Human Heart. Circulation 2020 aug;142(5):466-482. https://singlecell. broadinstitute.

[54] Prasad V, Lorenz JN, Lasko VM, Nieman ML, Huang W, Wang Y, et al. SERCA2 Haploinsufficiency in a Mouse Model of Darier Disease Causes a Selective Predisposition to Heart Failure. BioMed Research International 2015;2015.

[55] McMullen JR, Shioi T, Huang WY, Zhang L, Tarnavski O, Bisping E, et al. The Insulin-like Growth Factor 1 Receptor Induces Physiological Heart Growth via the Phosphoinositide 3-Kinase(p110 $\alpha)$ Pathway. Journal of Biological Chemistry 2004 feb;279(6):4782-4793. http://www. jbc.org.

[56] Cilvik SN, Wang JI, Lavine KJ, Uchida K, Castro A, Gierasch CM, et al. Fibroblast Growth Factor Receptor 1 Signaling in Adult Cardiomyocytes Increases Contractility and Results in a Hypertrophic Cardiomyopathy. PLoS ONE 2013 dec;8(12):e82979. https://dx.plos.org/10.1371/journal.pone.0082979.

[57] Gao S, Puthenvedu D, Lombardi R, Chen SN, Established and emerging mechanisms in the pathogenesis of arrhythmogenic cardiomyopathy: A multifaceted disease. MDPI AG; 2020. /pmc/articles/PMC7503882/ /pmc/articles/PMC7503882/?report=abstract https://www.ncbi.nlm.nih.gov/pmc/articles/PMC7503882/. 
[58] Lalem T, Zhang L, Scholz M, Burkhardt R, Saccheti V, Thiery J, et al. P830Cyclin dependent kinase inhibitor 1C (CDKN1C), a novel female-specific marker of left ventricular dysfunction after acute myocardial infarction. European Heart Journal 2018 aug;39(suppl_1). https://academic.oup.com/eurheartj/article/doi/10.1093/eurheartj/ehy564.P830/5084245.

[59] Zhou C, Li C, Zhou B, Sun H, Koullourou V, Holt I, et al. Novel nesprin-1 mutations associated with dilated cardiomyopathy cause nuclear envelope disruption and defects in myogenesis. Human molecular genetics 2017 jun;26(12):2258-2276. https://pubmed.ncbi.nlm.nih.gov/28398466/.

[60] Sandra M, Maria Pia L, Stefano C, Pietro P, Crociani P, Aldo R, et al. Emery-Dreifuss muscular dystrophy type 4: A new SYNE1 mutation associated with hypertrophic cardiomyopathy masked by a perinatal distress-related spastic diplegia. Clinical Case Reports 2019 may;7(5):10781082. /pmc/articles/PMC6509902/ /pmc/articles/PMC6509902/?report=abstract https://www.ncbi.nlm.nih.gov/pmc/articles/PMC6509902/.

[61] Shi X, Zhang Y, Li B, Peng M, Yuan Y, Wang X, et al. NOTCH4 is a possible novel susceptibility gene for dilated cardiomyopathy in the Chinese population: A case-control study. Journal of Clinical Laboratory Analysis 2018 jul;32(6):e22436. http://doi.wiley.com/10.1002/jcla. 22436.

[62] Ma S, Feng J, Zhang R, Chen J, Han D, Li X, et al. SIRT1 Activation by Resveratrol Alleviates Cardiac Dysfunction via Mitochondrial Regulation in Diabetic Cardiomyopathy Mice. Oxidative Medicine and Cellular Longevity 2017;2017:1-15. https ://www.hindawi.com/journals/omcl/2017/4602715/.

[63] Yan T, Huang J, Nisar MF, Wan C, Huang W, The beneficial roles of SIRT1 in drug-induced liver injury. Hindawi Limited; 2019. https://pubmed.ncbi.nlm.nih.gov/31354914/.

[64] Li Y, Wong K, Giles A, Jiang J, Lee JW, Adams AC, et al. Hepatic SIRT1 attenuates hepatic steatosis and controls energy balance in mice by inducing fibroblast growth factor 21. Gastroenterology 2014;146(2). https://pubmed.ncbi.nlm.nih.gov/24184811/.

[65] Bellet MM, Masri S, Astarita G, Sassone-Corsi P, Della Fazia MAD, Servillo G. Histone deacetylase SIRT1 controls proliferation, circadian rhythm, and lipid metabolism during liver regeneration in Mice. Journal of Biological Chemistry 2016 oct;291(44):23318-23329. https://pubmed.ncbi.nlm.nih.gov/27634039/.

[66] Lee JH, Song MY, Song EK, Kim EK, Moon WS, Han MK, et al. Overexpression of SIRT1 protects pancreatic $\beta$-cells against cytokine toxicity by suppressing the nuclear factor- $\kappa \mathrm{B}$ signaling pathway. Diabetes 2009 feb;58(2):344-351. https://pubmed.ncbi.nlm.nih.gov/19008341/.

[67] Cui L, Guo J, Zhang Q, Yin J, Li J, Zhou W, et al. Erythropoietin activates SIRT1 to protect human cardiomyocytes against doxorubicin-induced mitochondrial dysfunction and toxicity. Toxicology Letters 2017 jun;275:28-38. https://pubmed.ncbi.nlm.nih.gov/28456571/.

[68] Kalliora C, Kyriazis ID, Oka SI, Lieu MJ, Yue Y, Area-Gomez E, et al. Dual PPAR $\alpha / \gamma$ activation inhibits SIRT1-PGC1 $\alpha$ axis and causes cardiac dysfunction. JCI Insight 2019 sep;4(17). https://pubmed.ncbi.nlm.nih.gov/31393858/.

[69] Duisters RF, Tijsen AJ, Schroen B, Leenders JJ, Lentink V, Van Der Made I, et al. MiR-133 and miR-30 Regulate connective tissue growth factor: Implications for a role of micrornas in myocardial matrix remodeling. Circulation Research 2009 jan;104(2):170-178. https://pubmed.ncbi.nlm.nih.gov/19096030/ https://pubmed.ncbi.nlm.nih.gov/19096030/?dopt=Abstract.

[70] Miller TE, Ghoshal K, Ramaswamy B, Roy S, Datta J, Shapiro CL, et al. MicroRNA$221 / 222$ confers tamoxifen resistance in breast cancer by targeting p27Kip1. Journal of Biological Chemistry 2008 oct;283(44):29897-29903. https://pubmed.ncbi.nlm.nih.gov/18708351/ https://pubmed.ncbi.nlm.nih.gov/18708351/?dopt=Abstract.

[71] Cacheiro P, Muñoz-Fuentes V, Murray SA, Dickinson ME, Bucan M, Nutter LMJ, et al. Human and mouse essentiality screens as a resource for disease gene discovery. Nature Communications 2020 dec;11(1):1-16. https://doi .org/10.1038/s41467-020-14284-2. 
[72] Wishart DS, Knox C, Guo AC, Shrivastava S, Hassanali M, Stothard P, et al. DrugBank: a comprehensive resource for in silico drug discovery and exploration. Nucleic acids research 2006;34(Database issue). https://pubmed.ncbi.nlm.nih.gov/16381955/.

[73] Ursu O, Holmes J, Knockel J, Bologa CG, Yang JJ, Mathias SL, et al. DrugCentral: Online drug compendium. Nucleic Acids Research 2017 jan;45(D1):D932-D939. https://pubmed.ncbi.nlm.nih.gov/27789690/. 